\title{
Funcionalizar el Desacuerdo. Definición y Propuesta de Intervención Mediadora ante Negociaciones Atascadas
}

\section{Functional Disagreement. Definition and Mediative Intervention Proposal before Blocked Negotiations}

\author{
Miguel Hierro Requena \\ Ayuntamiento de Madrid, España
}

\begin{abstract}
Resumen. Desde mi experiencia como Mediador Familiar, propongo una definición de lo que considero una Negociación Atascada. A continuación, describo una serie de técnicas propuestas por otros autores para tratar de desatascar la situación. Finalmente, y desde el Problem Solving Estratégico, describo una técnica a la que denomino Funcionalizar el Desacuerdo, para ser utilizada en estas circunstancias.

Palabras clave: mediación familiar, negociación, técnica de mediación, funcionalizar el desacuerdo.

Abstract. From my experience as Family Mediator, I make a proposal of what I understand as a Blocked Negotiation. Next, I describe a group of actions proposed by other authors in order to solve those situations. Finally, from Strategic Problem Solving, I describe an action that I have named Functional Disagreement, to be used in such circumstances.

Key words: family mediation, negotiation, mediation action, functional disagreement.
\end{abstract}

Los procesos de cambio o crisis, parte natural del proceso evolutivo familiar, son inevitables. En la mayoría de las ocasiones, el Sistema Familiar encuentra una manera adaptativa de enfrentar el cambio. Esta adaptación facilita y permite que la infancia se torne en juventud y que la juventud alcance la edad adulta. Así mismo, los padres modifican paulatinamente la manera de cuidar y criar a sus hijos, desarrollando nuevas funciones y abandonando otras que dejan de ser necesarias o eficaces. En algunas ocasiones el proceso evolutivo se atasca, el cambio o crisis se convierte no en una situación temporal sino que adquiere una estabilidad que produce tensiones, malestares y conflictos en los miembros de la familia. Y en ocasiones llega la ruptura de la pareja, la salida del domicilio de un hijo adulto no

La correspondencia sobre este artículo debe enviarse al autor al email:mime_hierro@yahoo.es por acuerdo sin por conflicto, el enfrentamiento en torno a la gestión de una herencia... estas y muchas otras son ocasiones en las que la familia necesita tomar decisiones, de carácter más o menos estable, que faciliten la gestión de las consecuencias de un conflicto o crisis.

Los servicios, espacios y procesos de Mediación Familiar esperan ser facilitadores de una comunicación efectiva para transmitir necesidades y hacer propuestas que, de llevarse a cabo, cubran la totalidad o una parte esas necesidades, y alcanzar acuerdos en torno a algunas de esas propuestas. Esta facilitación surge desde un contexto neutral, que facilita la disminución de la intensidad emocional resultante de las dificultades familiares, y propiciando un ambiente donde la comunicación resulte más eficaz y colaborativa.

Todos los agentes implicados en la Mediación Familiar: usuarios, técnicos, profesionales y respon- 
sables de los programas o servicios correspondientes partimos de la anterior premisa. Sin embargo, nos son escasas las ocasiones en que la negociación se atasca.

\section{La Negociación Atascada}

En este trabajo propongo utilizar el término Negociación Atascada así como describir una intervención que puede resultar eficaz para disolver dicha situación. Denominaré Negociación Atascada a la relación que se establece dentro del espacio o sesión de mediación entre ambos ${ }^{1}$ usuarios y el mediador con cada uno de ellos y los dos al tiempo. Es decir, tanto entre el padre y la madre de la familia que buscan acuerdos, como entre el mediador y la madre, el mediador y el padre o el mediador y el sistema parental.

La Negociación Atascada surge en un proceso de mediación en marcha, en el que han tenido lugar al menos una conversación sobre las condiciones del proceso de mediación, los objetivos del mismo y las necesidades que se pretende tratar de resolver, y se ha obtenido un consentimiento y acuerdo entre los usuarios y el mediador al respecto. No se trataría de dificultades en la construcción del Espacio de Mediación. La Negociación Atascada no está relacionada con la desconfianza entre los miembros de la familia o las dudas sobre la finalidad de las conversaciones con el Mediador. Entiendo que este tipo de dificultades han de resolverse de manera previa al inicio de la negociación. Para que el Espacio de Mediación tenga más posibilidades de resultar efectivo, antes de iniciar la recogida de propuestas y búsqueda de alternativas ya deben haberse resuelto estas dudas.

Así mismo, para entender que se trata de una Negociación Atascada debe haberse concretado un aspecto, actualmente falto de acuerdo entre el padre

\footnotetext{
1 En mi experiencia, lo más frecuente es que en la sesión de Mediación Familiar estén presentes dos usuarios. No obstante, en ocasiones el encuentro tiene lugar con más personas (usuarios o profesionales). A lo largo del texto haré referencia a encuentros entre un mediador y dos usuarios del servicio, padre y madre de una familia en proceso de divorcio, que buscan un acuerdo útil para ambos. Las conclusiones que alcanzo al final son, en mi opinión, igualmente aplicables a sesiones de comediación, sesiones con más de dos miembros de la familia, etc.
}

y la madre, y cada uno de ellos haber ofrecido una (o más) propuestas de acuerdo que, de alcanzarse, entienden que permitiría a toda la familia un funcionamiento más eficaz para todos.

Aunque no es el objetivo de este artículo, señalar que muchas de las dificultades descritas por mediadores y usuarios para completar de manera efectiva el Proceso de Mediación Familiar están íntimamente relacionadas con un deficitario subproceso de Pre - Mediación Familiar. El acuerdo entre todos los agentes participantes sobre las condiciones, premisas y objetivos del Proceso de Mediación es fundamental para que éste salga adelante.

La Universidad de Harvard propuso los principios básicos de un proceso de negociación que promueve el beneficio mutuo y se aleja de la negociación dura donde se persigue conseguir lo que se pretende incluso a costa del otro, y la negociación blanda donde se evita el enfrentamiento aun a costa de olvidar los intereses propios. Esta negociación colaborativa trataría de:

1.- Separar a las personas del problema con la finalidad de reconducir y disminuir las respuestas emocionales y relacionales

2.- Centrarse en los intereses y no en las posiciones con la finalidad de abrir el abanico de alternativas que cubran una necesidad.

3.- Recurrir a criterios objetivos para tratar de despersonalizar el conflicto y establecer algunos criterios ${ }^{2}$.

4.- Crear alternativas que cubran las necesidades de ambas personas y que ofrezcan un beneficio mutuo.

La Negociación Atascada tiene lugar principalmente en el momento de Recogida de propuestas y Negociación o, con carácter más excepcional, en el momento de la Definición de Acuerdos (ver figura 1).

La Negociación Atascada se caracteriza por, tras la definición de una necesidad familiar (o de uno de los miembros de la familia) y la recogida de al menos una propuesta para cubrir dicha necesidad procedente de cada uno de los usuarios, la incompa-

\footnotetext{
${ }^{2}$ En Procesos de Mediación Familiar, la búsqueda de criterios objetivos puede ser especialmente complicada dado el carácter subjetivo que tiene la expectativa de cada persona sobre lo que es y espera de una familia.
} 
Figura 1. Proceso de mediación familiar



tibilidad de las propuestas alcanzadas y la inmovilidad de ambos usuarios bien hacia el desarrollo de otras propuestas bien hacia la aceptación de alguna de las propuestas surgidas del otro miembro de la familia.

Esta inmovilidad no estaría relacionada con la desconfianza, el despecho etc. Estaría relacionada con la percepción por parte de cada uno de los usuarios de que las propuestas que está descartando (es decir, las que provienen de la otra parte) no satisfacen de manera adecuada su propia necesidad. Recordemos aquí que las necesidades familiares son las necesidades de los miembros de la familia, y que el conflicto familiar surge precisamente de la manifestación de necesidades incompatibles entre unos y otros.

Carolina y David han puesto en marcha, junto con un profesional de un servicio público municipal, un Proceso de Mediación Familiar con la finalidad de alcanzar acuerdos para organizar su situación familiar tras el divorcio. Han estado hablando de los gastos de toda la familia (cada uno de ellos y sus dos hijos) así como de los ingresos que tienen entre ambos. Carolina ha propuesto que David ingrese en una cuen- 
ta corriente a su nombre la cantidad de trescientos cuarenta euros, ciento setenta por cada uno de sus hijos. David, que está de acuerdo en cubrir entre ambos los gastos de los niños y que sea él quien haga un ingreso en una cuenta a nombre de Carolina, propone que dicho ingreso sea de doscientos noventa euros. Tras realizar un Caucus (Bolaños, 2008) individual con cada uno de los usuarios, el mediador llega a la conclusión de que Carolina piensa que, con una aportación menor de trescientos cuarenta euros no podrá asumir con razonable seguridad los gastos corrientes de ambos hijos. Por otro lado, David plantea que no podrá asumir la aportación además de cubrir sus gastos personales con una aportación por encima de los doscientos noventa euros.

La situación de Carolina y David sí entraría en la definición de Negociación Atascada, puesto que ambos escuchan y aceptan por válido el punto de vista del otro y su propuesta, pero entienden que no cubre adecuadamente las necesidades familiares percibidas.

Paloma y Ramón llevan dos años separados. La separación fue contenciosa, estableciéndose en una sentencia de separación que sus hijos pasasen fines de semana alternos con uno y otro. Recientemente, Ramón y Paloma acudieron a un Servicio de Mediación Familiar puesto que Ramón ha solicitado ampliar los fines de semana, de forma que abarquen desde el jueves hasta el martes. Paloma no cree necesario hacer ninguna modificación en este aspecto.

Tras hablar con ellos en un Caucus, Ramón explica que está muy a gusto con sus hijos y no entiende porqué deben pasar mucho más tiempo con su madre que con él. Su propuesta trata de atajar su necesidad de pasar más tiempo con sus hijos. Paloma, por su parte, explica que es evidente que Ramón hace esta propuesta con la finalidad de justificar una reducción de la cantidad mensual que aporta para los gastos de sus hijos. Paloma piensa que Ramón nunca ha mostrado verdadero interés por sus hijos. Desde el punto de vista de Paloma, la propuesta de Ramón responde a la "necesidad" de asumir menos gastos.

La situación de Paloma y Ramón no entraría dentro de la definición de Negociación Atascada, puesto que perciben la necesidad de manera distinta. No hay acuerdo sobre la necesidad a cubrir y en consecuencia las propuestas parecen incongruentes. Antes de entrar a valorar propuestas o negociar, habría que dedicar más tiempo a poner en común las necesidades, legitimarlas y proponer el espacio como un momento legítimo donde hacer propuestas. En caso de seguir adelante sin este trabajo previo (premediación), la relación coparental podría resentirse. Si no existe acuerdo sobre la necesidad en torno a la cual negociaremos propuestas, como mediador propondría finalizar el Servicio entendiendo que no hay un acuerdo sobre las condiciones del mismo.

Existen una serie de técnicas a disposición del mediador para facilitar un acuerdo en premediación que permita iniciar la búsqueda y negociación de alternativas. Las premisas, para iniciar la fase de Mediación, pasarían por acordar:

- Las decisiones corresponden a los dos usuarios y la neutralidad del Mediador, que no realizará juicios ni valoraciones más allá de los relacionados con el Proceso de Mediación.

- Las necesidades familiares a cubrir, pudiendo cada uno de los usuarios proponer cuales cree que deberían atenderse, pero atendiéndose solamente aquellas que ambos acuerden ${ }^{3}$.

- La confidencialidad del Espacio de Mediación, especificándose que el Mediador nunca podrá emitir informes ni participar como testigo ante tribunales ${ }^{4}$.

La voluntariedad del servicio no creo tan relevante especificarla, pudiendo ponerse sobre la mesa si en algún momento se percibe en alguno de los usuarios indicadores de que se siente obligado a participar.

\section{Qué hacer ante Negociaciones Atascadas}

Ante la Negociación Atascada ya existen diversos autores que han desarrollado propuestas al respecto. Ignacio Bolaños (2008) habla de la fase del Proceso de Mediación en que el Mediador promue-

\footnotetext{
${ }^{3}$ Para que el Proceso de Mediación ofrezca un resultado óptimo, los usuarios deben QUERER alcanzar ese resultado. En mi experiencia, ha sido muy frustrante trabajar con familias donde uno de los usuarios quería, mientras que el otro se limitaba a sabotear el trabajo del Mediador.

${ }^{4}$ Fundamental en procesos ya judicializados, donde tanto los usuarios como sus abogados pueden ver en el Mediador una fuente de información para el tribunal. No es extraño confundir al mediador bien con un perito, bien con un técnico del equipo psicosocial del juzgado.
} 
ve la creación de opciones y alternativas. Partiendo de un lenguaje que hable de la posibilidad de hacer y se aleje de exigencias o reproches, propone que el Mediador pueda facilitar:

- El encadenamiento de ideas alternativas o complementarias para atajar la necesidad.

- La búsqueda de nuevas soluciones distintas de las iniciales o de las habituales.

- Aportaciones constructivas que se manifiesten de manera concreta y aplicable a la realidad familiar.

- La normalización de la dificultad como parte del proceso de decisión.

En la página 184, Bolaños habla de una intervención paradójica que normalice el derecho de las partes a bloquear la toma de decisiones. Está propuesta está íntimamente relacionada con la que plantearé más adelante.

Trinidad Bernal (2002) expone un listado de preguntas que el Mediador puede realizar a los miembros de la familia para obtener nuevas propuestas o alternativas distintas de las iniciales. En la página 183 menciona:

- Las preguntas Creativas, incluyendo en este grupo las preguntas hipotéticas; ¿Cómo lo llevarías a cabo? y preguntas estimulantes; ¿existen otras maneras de solucionar esto?

- Las preguntas reconductoras, que pretenden desviar la interacción improductiva de la familia; ¿a qué os lleva esto? Si no surgen otras posibilidades ¿con qué situación os encontraréis?

Marla Isaacs, Braulio Montalvo y David Abelsohn (2009), aunque su texto está dirigido a terapeutas y no a mediadores, proponen, con la finalidad de generar alianzas entre personas a enfrentadas, la elaboración de un discurso elaborado y amplio por parte del técnico (en nuestro caso del Mediador) donde se enumeren con sorpresa tanto las inquietudes comunes, como las virtudes parecidas. Cuando la negociación se atasca el Mediador puede tomar la palabra, dedicar unos minutos a enumerar todos los aspectos que a ambos preocupan y las necesidades comunes que se han detectado, elogiar el esfuerzo que a ambos les supone acudir al Espacio de Mediación para tratar de facilitar la transición familiar y finalizar transmitiendo que, si bien es un proceso difícil, el Mediador es de la opinión de que es posible encontrar una vía válida para ambos. En este discurso es interesante incluir ejemplos de anécdotas que la familia haya podido ir dejando caer en las reuniones. A continuación se retomaría la negociación dando por hecho que surgirán nuevas alternativas ...aparte de las posibilidades que ya hemos barajado ¿qué otras podríamos poner sobre la mesa que quizás faciliten las cosas?

\section{Propuesta de intervención - premisas y justificación}

Es una premisa fundamental, para la aplicación de la técnica propuesta, que la Premediación se haya realizado de manera correcta. En aquellos casos en que estén interviniendo otros profesionales previamente al inicio del Proceso de Mediación, el Mediador debe contactar con ellos, presentarse, solicitar apoyo al proceso que se inicia y ofrecerse para conversaciones futuras. La finalidad de estos contactos es que el máximo número de personas posibles implicadas en el proceso de ruptura de pareja sepan del Proceso de Mediación y traten de facilitarlo.

Así mismo, otra premisa relevante es tratar de aplicar las técnicas descritas más arriba (u otras que el Mediador tenga a su alcance) cuando la búsqueda y construcción de alternativas y posible soluciones va teniendo lugar. No esperemos a que se produzca el atasco, tratemos de prevenirlo. Como Mediador, soy de la opinión de que la legitimación de las necesidades, propuestas y actitudes es una herramienta de vital importancia. Taylor (1988) hace hincapié en la función que desarrolla el Mediador para devolver la realidad a parejas separadas. Somos Agentes de Realidad.

Jesús.-Una vez separados, no veo mucho sentido a mostrarnos distantes. Entiendo que puedes venir a mi casa a pasar la tarde con los niños, ayudarles con los deberes... y yo haré del mismo modo en tu domicilio.

$M^{a}$ del Mar.-pero jcómo vamos a estar entrando y saliendo el uno de la casa del otro! ;Si ahora vivimos juntos y no nos dirigimos la palabra!

Jesús.-bueno, pues hablando mucho...v vale que firmemos algunos acuerdos, pero luego podemos hacer lo que consideremos. 
$M^{a}$ del Mar.-Ya... pero quizás yo consideraré que tú no vengas a mi casa.

Jesús.-Pues chica, no se como esperas entonces que las cosas vayan bien.

Mediador-Entiendo que Jesús está preocupado por el cambio, y pone mucho interés en que las cosas sean lo más normales posibles. Mar, por su parte, plantea que esto puede generar inconvenientes. Puede ser útil recordar que os estáis separando, principalmente, por las dificultades que os supone hablar y coordinaros.

Jesús.-Pues no entiendo porque tiene que ser tan difícil.

Mediador-Sencillamente porque os estáis separando. Y muchas parejas, cuando se separan, encuentran cierta tranquilidad en verse menos que cuando estaban juntos.

Esta conversación la mantuve con Jesús y $\mathrm{M}^{\mathrm{a}}$ del Mar, pareja que aún convivía, con un nivel de conflicto doméstico muy alto y una comunicación de pareja muy deteriorada (como ambos me habían descrito anteriormente) La intervención final subraya la poca coherencia de la idea de Jesús, idea que además no compartía $\mathrm{M}^{\mathrm{a}}$ del Mar, con la situación familiar actual.

Otra técnica que ofrece resultados muy llamativos y que quizás no utilizamos todo lo que debiéramos, probablemente porque resulta engorrosa, es el Caucus. El Caucus (separar a los miembros de la pareja durante un periodo de tiempo concreto para poder hablar individualmente con cada uno de ellos) ha sido descrito como Caucus por Bolaños (2008) y como Reuniones individuales por Bernal (2002). Algunos autores (Taylor, Folberg, Pruitt) han mencionado que el Caucus puede generar dificultades por la percepción que se puede generar en los usuarios de pérdida de imparcialidad del Mediador, ansiedad al desconocerse lo que la otra persona ha dicho o se le ha dicho... Si se sigue la propuesta de Bolaños, realizando el Caucus (o reunión individual) en tras fases; una primera de escucha activa y fuerte empatización del Mediador hacia el usuario, una segunda para retomar la necesidad y cuestionar si la postura adoptada realmente cubrirá dicha necesidad y una tercera para proponer acercar la postura a la del otro usuario, entonces la posibilidad de que esta técnica no facilite un Proceso de Mediación más satisfactorio disminuye notablemente.

En ocasiones, todo lo escrito más arriba no es sufi- ciente. Y, después de mucho esfuerzo por parte de usuarios y mediadores, nos encontramos de nuevo con la Negociación Atascada por la falta de movilidad de cada una de las personas en su postura.

Para construir mi propuesta, recurrí al texto de Giorgio Nardone (2009) Problem solving estratégico. En este texto el autor propone una serie de pasos para resolver problemas a los que nos enfrentamos las personas y que, aparentemente, no disponemos de una solución. En su momento, me limité dejarme guiar por las propuestas de Nardone:

\section{Definir el problema}

Ambos usuarios del Servicio de Mediación se muestran inmovilistas ante la búsqueda o aceptación de propuestas distintas de la que entienden como más oportuna.

\section{Determinar y acordar el objetivo}

Mi objetivo es facilitar que alcancen un acuerdo al respecto. El cambio concreto surgirá porque uno y otro dirán que aceptan como válida una opción que coincidirá. Esto me permitirá bien pasar a otro tema del Proceso de Mediación o bien finalizarlo.

\section{Estudiar los intentos de solución llevados a cabo}

He tratado, de diferentes maneras, de promover nuevas propuestas.

También he tratado, principalmente individualmente, de facilitar que acepten la propuesta de la ex pareja.

\section{Como empeorar la situación}

Para que el Proceso de Mediación se atasque más, creo que principalmente puedo insistir en que no hablaremos de otra cosa hasta que no haya un acuerdo en este aspecto. Así mismo, puedo tratar de advertir de las funestas consecuencias que traerá a su familia su actitud. Por último, podría tratar 
de señalarles como culpables o responsables de esas consecuencias. Además podría tratar de juzgarles y transmitir que si fueran personas maduras y responsables harían un esfuerzo por alcanzar un acuerdo.

\section{El escenario más allá del problema}

Cuando el objetivo se haya cumplido completamente, como Mediador dedicaré mis esfuerzos a atender a otras familias. La familia, por su parte, funcionará de manera razonablemente adecuada con las decisiones que el padre y la madre han alcanza$\mathrm{do}^{5}$.

\section{Cuales serían los primeros pequeños pasos que nos acerquen al objetivo ${ }^{6}$}

Quitar tensión a la posibilidad de no alcanzar un acuerdo, relajar la comunicación.

\section{La técnica del escalador: situarnos en el objetivo establecido y enumerar los pasos a la inversa hasta el momento actual}

4 - alcanzan un acuerdo al respecto.

3 - menciono que parecen estar cerca del acuerdo.

2 - uno, otro o ambos varían su postura.

1 - propongo pasar a otro tema, normalizo que exista esta disputa y que probablemente no será la única, después de todo, las personas divorciadas tienen diferencias. Señalo que no ponerse de acuerdo en este aspecto no solo no implica falta de responsabilidad, sino que probablemente supone un alto ejercicio de responsabilidad (sería más fácil aceptar cualquier cosa y luego no cumplirla) y que probablemente yo no he entendido bien el efecto protector

\footnotetext{
${ }^{5}$ Escribiendo este párrafo, comprendí que alcanzar un consenso en este punto y la posible firma de un Acuerdo de Mediación que lo incluya no implica necesariamente un funcionamiento óptimo. Es decir, es posible (incluso probable) que antes o después la familia se encuentre con dificultades para funcionar como padre y madre divorciados.

${ }^{6}$ Esta fue la parte del ejercicio que más dificultades me generó ¿Cuáles podrían ser esos primeros pasos, distintos de los ya intentados...?
}

que mantener su postura cada uno de ellos tiene sobre todos los miembros de la familia.

0 - Ambos usuarios del Servicio de Mediación insisten en que no pueden aceptar las propuestas o iniciativas del otro.

La anterior secuencia, y especialmente el paso 1, coincide con los principios sistémicos descritos por Fish, Weakland y Segal, en su trabajo La táctica del cambio (1994). En este texto describen de manera eficaz y convincente como los sistemas se organizan en torno a las soluciones ineficaces. De modo que, entendiendo el Sistema Mediacional como el formado por ambos usuarios y el Mediador, la solución ineficaz al problema consistiría en insistir (de diferentes maneras, técnicas etc) en la necesidad de que cedan y alcancen un acuerdo. Yo, como Mediador, estaría insistiendo y presionando a ambos, y cada uno de ellos al otro. Un atasco interesante.

En La táctica del cambio se propone, ante sistemas organizados en torno a soluciones ineficaces, tratar de influir sobre el sistema para cambiar el intento de solución. Un cambio, a ser posible, notablemente distinto. De modo que el mensaje "os entiendo, haced un esfuerzo por entenderos y poneros de acuerdo por el bien de vuestra familia" puede fácilmente identificarse como solución ineficaz, y serviría como solución alternativa "no he conseguido entenderlo del todo, está bien que mantengáis el desacuerdo porque algo debe aportar a vuestra familia."

De nuevo, esta propuesta, aunque quizás no es idéntica, sí se asemeja notablemente a la propuesta descrita por Bolaños:

Una intervención aún más paradójica supone normalizar el derecho de las partes a bloquear la toma de decisiones, proporcionando una razón para la indecisión. La paradoja legitima el derecho a la ambivalencia y proporciona el control para cambiar. (Bolaños, 2008, Pág. 184).

El ejercicio y reflexión que descrito en este artículo lo desarrollé personalmente durante algunas semanas hace aproximadamente dieciocho meses. Desde entonces he aplicado esta técnica en trece casos de Mediación Familiar, siempre relacionada con la ruptura de pareja. Así mismo, siempre recurrí a esta técnica después de haber intentado (y fracasa- 
do) facilitar el cambio con, si no todas, algunas de las propuestas de otros autores y descritas más arriba. De los mencionados trece casos, nueve de ellos alcanzaron un acuerdo al respecto de la necesidad identificada y aceptada por ambos en la que se había atascado la negociación. Señalar que dicho acuerdo no siempre llegó a continuación de la intervención. En algunas ocasiones, se acordó el desacuerdo, y se pasó a buscar propuestas para resolver otra necesidad. Pero fueron los usuarios, en el momento de la redacción del Acuerdo de Mediación quienes, por iniciativa propia, retomaron brevemente y acordaron una decisión.

\section{Técnica propuesta:}

\section{FUNCIONALIZAR EL DESACUERDO}

\section{Proponer pasar a otro tema, normalizar} que exista esta disputa y que probablemente no será la única; después de todo, las personas divorciadas tienen diferencias.

2. Señalar que no ponerse de acuerdo en este aspecto no solo no implica falta de responsabilidad, sino que probablemente supone un alto ejercicio de responsabilidad: sería más fácil aceptar cualquier cosa y luego no cumplirla.
3. Reconocer que probablemente no hemos entendido bien el efecto protector que mantener su postura cada uno de ellos tiene sobre todos los miembros de la familia.

4. Preguntar ¿de qué otra necesidad familiar os gustaría hablar ahora?

Espero seguir teniendo la oportunidad de aplicarla. Así mismo, animaría a otros mediadores a aplicarla, tal y como la describo o con modificaciones o adaptaciones que estimen oportunas, para corroborar o descartar su utilidad.

\section{Referencias}

Bernal, T. (2002). La Mediación. Madrid: Colex. Bolaños, I. (2008) Hijos Alineados Y Padres Alienados. Madrid: Reus.

Isaac, S., Marla, B., Montalvo, B. Y Abelsohn, D. (2009). Divorcio Difícil. Buenos Aires: Amorrortu.

Nardone, G. (2009). Problem Solving Estratégico. Barcelona: Herder.

Taylor, A. (1988). A General Theory of Divorce Mediation. En J. Folberg y A. Miles, (Eds.) Divorce Mediation, Theory And Practice (pp.6182). New York: The Guilford Press.

Manuscrito recibido: 25/01/2012

Revisión recibida: 16/04/2012

Aceptado: 19/04/2012 